\title{
A Framework for the Creating, Expressing and Sharing of User's Emotion
}

\author{
Kyoung Shin Park ${ }^{1}$, Yongjoo $\mathrm{Cho}^{2}$ and Dong Keun $\mathrm{Kim}^{2}{ }^{*}$ \\ ${ }^{1}$ Department of Multimedia Engineering, Cheonan, Chungnam, Korea \\ ${ }^{2}$ Department of Digital Media, Sangmyung University, Seoul, Korea \\ ${ }^{1}$ kpark@dankook.ac.kr, ${ }^{2}\{y c h o, d k i m\} @ s m u . a c . k r$ \\ Abstract
}

\begin{abstract}
This paper presents the Emotionally Intelligent Contents (EIC) framewyork designed to support the rapid development of interactive emotion antents. The EIC framework consists of the Emotion Recognizer for detecting user's emotional states, the Emotion Server for storing and sharing emotion data, the Emotion Content Authoring Tol for customizing user's own emotional expression rules, the Emotion Rule Controllex for processing user's emotional rules, and the Emotion Content Player for displaying the Content according to the user's emotional states and his/her personalized-emotion rules. This framework allows a designer to create the emotion content that interprets $\checkmark$ user's emotional state and adapts its visual and aural characteristics based on user-specified emotion rules. It dynamically alters userspecified images or models to express user emotions. It also enables users to share their emotion with others using customized emotion expressions applied in the content. Several prototype EICs were built with this framemork. A pilot study was conducted to compare the EIC against the original content and the initial result showed that the EIC had significantly more influence on the subject's emotion than the original content.
\end{abstract}

Keywords: Emotion interaction, Emotion recognition, Emotion contents, Emotion framework

\section{Introduction}

Affective computing technology, which adapts interaction to user emotional states, can significantly enhance the usage of the human-computer interaction system. Understanding and responding to user's emotions provides considerable advantages in various entertainment applications. The affective applications react in a friendly manner according to the users' needs and preferences [1]. Emotion recognition systems using speech, gesture, facial expression and physiological signals have been extensively investigated to achieve an enriched emotional interaction [2-4]. Emotional expression systems such as emotion facial animations, emotion avatars and emotion robots, have also been developed to present human-like emotion expression [5-8]. In addition, some efforts were made to allow for the sharing of emotional experiences among users through multimedia contents and applications $[9,10]$.

However, such emotion systems and applications were built with designer-defined components and several individual software libraries to meet the necessities of different service purposes. The emotion applications built with the encompassing libraries would

* Corresponding Author 
be difficult and time-consuming because the libraries were created separately and might not necessarily have been designed to work together. In order to support interoperability and efficient reusability of each component of the interactive emotion contents, a more general emotion interaction framework needs to be developed. The emotion interaction framework would support building blocks integrating emotion recognition, generation, and expression [10]. Some frameworks have been proposed, which recognize the user's emotional states so as to provide users with appropriate assistance in a timely and efficient manner [11]. Some existing architectures and libraries that provide emotion recognition use mono-modal or bimodal approaches [12].

Emotional interaction plays a critical role in multimedia contents such as movies, games and pictures. The primary goals of people enjoying such emotion contents are to be entertained and/or emotionally immersed in the contents. There have been many attempts to create such emotion contents. Kim and her colleagues developed an emotion content that allows users to add their own emotion characters, which explicitly respond to changes in user emotional states in real time [8]. Zhao ef' al studied emotion-driven interactive digital storytelling. They edited existing tootage of the TV show, Ugly Betty, and used the audience's emotions as the driving force for the narratives [13]. Roberts et' al used pre-authored text with pre-selected video clips to create an interactive storytelling experience [14]. In thrs study the user is asked to answer questions between iterations and, depending on the answer, is directed to different narratives. In this research, movies were used to strmulate user's emotions. Jones and Sutherland developed an emotionally responsive computer game [15]. They analyzed the player's speech to recognize bis/her emotional states, which was then used to change the physical or behavioral attributes of the game character. For example, if the player felt happy, the game-character became more active, whereas if the player found the game boring, the character became less energetic.

In order to create more immersed oreffective emotion contents, we consider that the emotion content should not only tespond to user emotion, but should also have the following requirements:

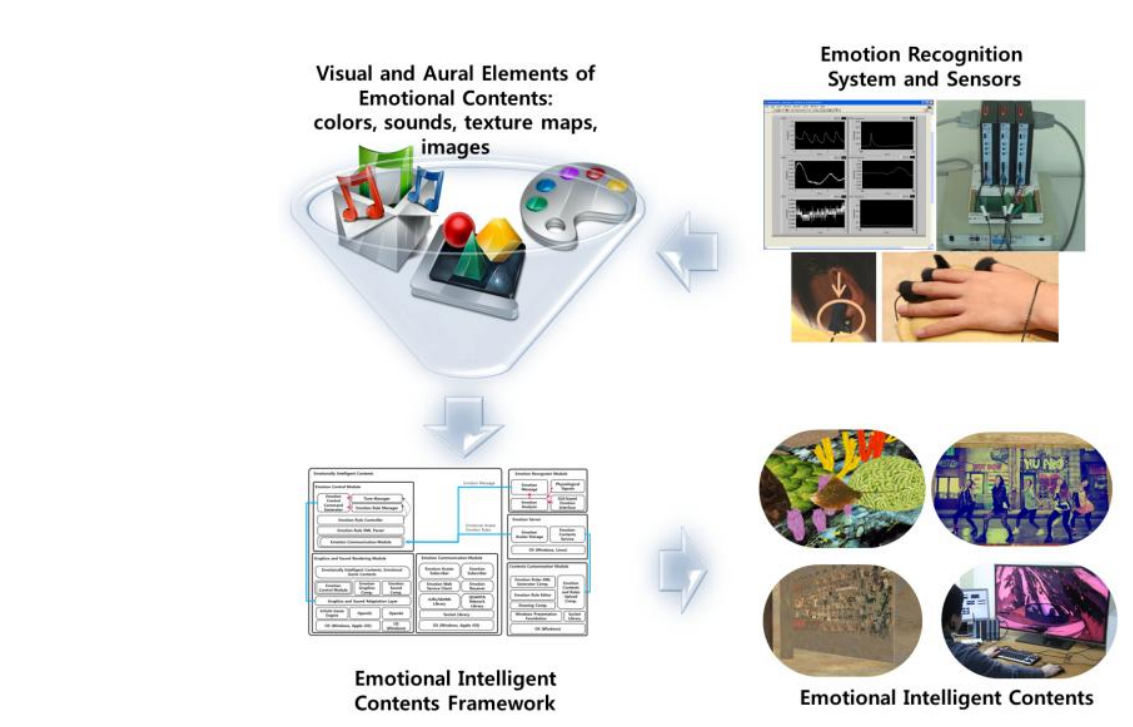

Figure 1. Conceptual Diagram of Emotionally Intelligent Content (EIC) 
- Provide dynamic changes in the emotion theme in real-time according to the user's emotional feelings.

- Support changes in the contents based on individual preferences.

- Define how the contents would be changed based on individual's customized emotion rules.

These requirements were applied to design our framework. Figure 1 shows the conceptual diagram of the Emotionally Intelligent Content (EIC) framework. The EIC framework is designed to facilitate the prototyping of emotion contents. The EIC refers to the content that goes beyond simple pre-defined emotion themes. EICfrocuses on allowing users to visually and aurally express or change the contents in real-time based on user emotional states. It uses the user's own emotion rules to definn how the visual and aural properties of the content (such as color, brightness, light, and sound) would be presented. In other words, users can design the properties of the emotion content based on their own preferences. Therefore, even if users see the same EIC, they may feel differently according to their own emotion rules. Conversely, their emotion may also be affected by manipulating the EIC.

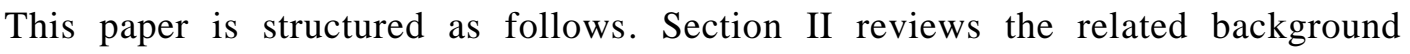
information about the emotion model and recognition method and some terminologies used in this research. Section III presents the system architecture of the EIC framework and its core modules. Section IV describes Emotionally Intelligent Content prototypes. Section V presents an efaluation of the emotion contents in terms of the user's emotional state. Section Vl presents conelusions and future research directions.

\section{Emotion Model and Emotion Recognition}

This section briefly describles the emotion model and the emotion recognition method to provide background information and to clarify some terminologies used in this research.

\subsection{Emotion Model}

Emotion recognition is an interesting but difficult issue. For theoretical or practical reasons, researchers have classified human emotions into discrete categories or on a dimensional basis. According to [16], user emotion is categorized into one of six basic emotion categories (derived from Darwin's pioneering work): anger, disgust, fear, joy, sadness, and surprise. On the other hand, emotion has also been characterized as labels on a two-dimensional coordinate system. The dimensional model of emotion representation allows the computation of continuous changes of user emotion using physiological measurements of affective states [17]. 


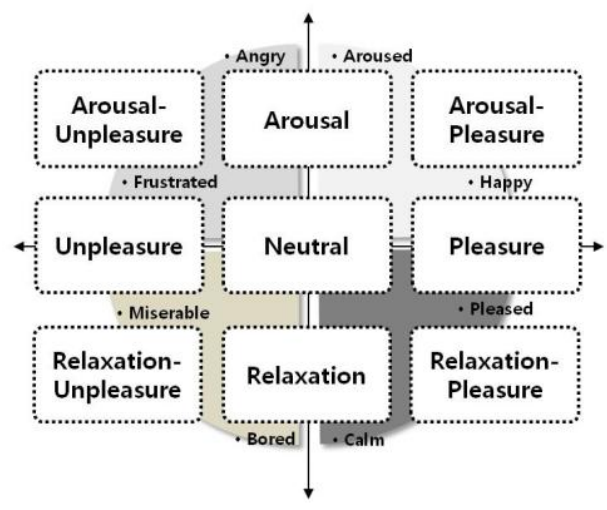

Figure 2. Heuristic 9-Domain Emotion Models

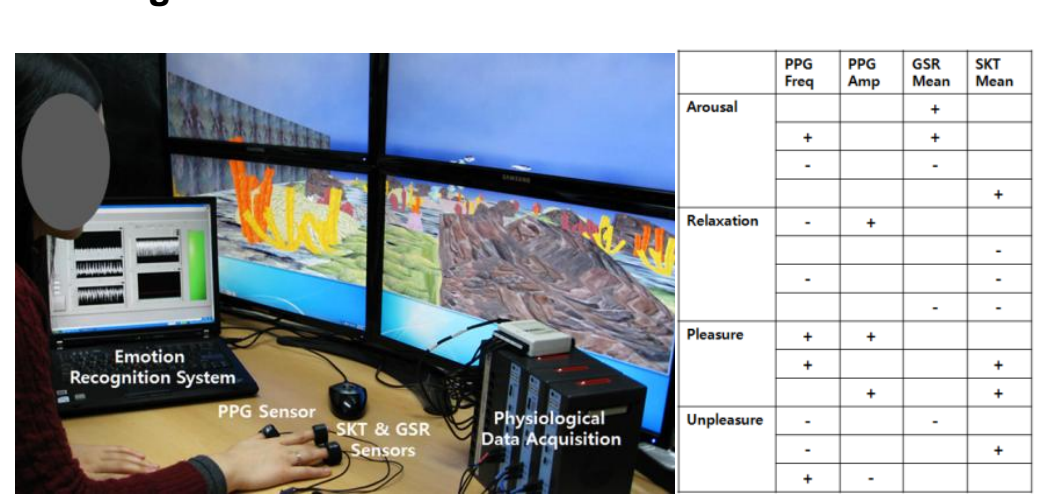

Figure 3. The EmotionaPnterface and the Emotion Recognition System using ANS(Autoriomous Nervous System) Physiological Signals

There are several dimensionar models of emotion that have been developed for emotion classification. For our study, we have adopted Russell's emotion model [18]. As shown in Figure 2, the two dimensions are represented by a vertical arousal axis and a horizontal valence axis. This two-dimensional emotion model, which we have adopted, is used to classify the current user's emotional state into nine categories based on continuous physiological changes and the measurement of affective states. This model targets nine regions of emotion. Pleasure, Arousal-Pleasure, Excitement, Arousal, Distress Misery, Depression, Sleepiness, and Contentment [10]

\subsection{Emotion Recognition}

In previous affective computing research, a variety of emotion recognition technologies, such as speech analysis, facial affect detection, body gesture/posture and physiological signal monitoring, have been used as a means of detecting a particular emotional state of the user [3]. In particular, emotion detection and recognition using physiological signs, such as Blood Pressure Pulse and Galvanic Skin Response, have shown promising results in cases where user affective states are directly related to changes in bodily signals. According to previous research [9, 16, 17], physiological monitoring based on the ANS (Autonomous Nervous System) is a reliable representation of true emotion, which cannot be consciously controlled due to its involuntary nature. Also, the emotional states retrieved from physiological data are very descriptive and easy to measure. ANS (Autonomous Nervous System) responses can be processed in real time to capture indirect perceptual emotion responses. 
Figure 3 shows the configuration of our emotion recognition system based on ANS (autonomous nervous system) physiological sensors, including PPG, SKT, and GSR. PPG sensors are often placed on the user's finger and earlobe to measure the cardiac synchronous cardiac changes in the blood volume. Changes in the amplitudes of PPG signals are, for humans, related to the levels of tension [3, 17]. SKT measures the thermal response of human skin. It is known that anger induces a large increase in skin temperature, whereas fear and sadness induce lower variations. GSR is used to measure when the skin momentarily becomes a better conductor of electricity in response to internal or external stimuli that are physiologically arousing. Major magnitude changes in GSR signals are related to emotional excitement and dynamic activity [17, 19]• In this system, the PPG frequency and amplitude and the SKT and GSR averages are extracted using a $200 \mathrm{~Hz}$ sampling rate and analyzed to estimate the corresponding emotion based on our heuristic model [20].

\section{Emotionally Intelligent Content Framework}

The Emotionally Intelligent Content (EIC) framework aims to create, express and share user's emotions depending on the user's preference for emotion expression. This framework is designed for easy construction of emotion contents customized for each individual and for sharing these contents with other users based on their own unique emotion rules. The emotion rules describe how the contents are rendered visually and aurally depending on the user's emotional state. The color properties are used to set foreground and background color, light, and brightness of objects in the erbotion contents. The aural properties are used to alter background music and sound effects of the contents. If there is no emotion rule specified by the user, default rules are used instead.

The default emotion rules are based on the survey results of the fashion industry's color theory study [21]. According to thrs study, people tend to get more pleasure when brighter colors are shown and the dontrast and saturation of colors are increased. Also, people seem to be more aroused yhen colors with increased luminosity are shown. Music and sound effects can be played differently in terms of altering sound speed and volume pitch based on the user's emotion. The Emotionally Intelligent Content framework consists of several core modules: the Emotion Recognizer, the Emotion Server, the Emotion Content Authoring Module, the Emotion Rule Controller Module, and the Emotion Content Player Module.

\subsection{The Emotion Recognizer and the Emotion Server}

As shown in Figure 4, the user's emotional states are identified by the Emotion Recognizer Module and then stored in the Emotion Server [8]. User emotion is classified as one of nine different states in a two dimensional space based on our heuristic nine-domain emotion model, which we have adopted from Russell's emotion model [8, 18]. The Emotion Recognizer Module is written in LabView utilizing BIOPAC MP100 system's PPG (Phtoplethsmography), SKT (Skin Temperature), and GSR (Galvanic Skin Response) sensors connected to a desktop computer. BIOPAC provides accurate and reliable data but it works with cable tethering. Hence a mobile prototype via a Bluetooth is also provided [9]. 


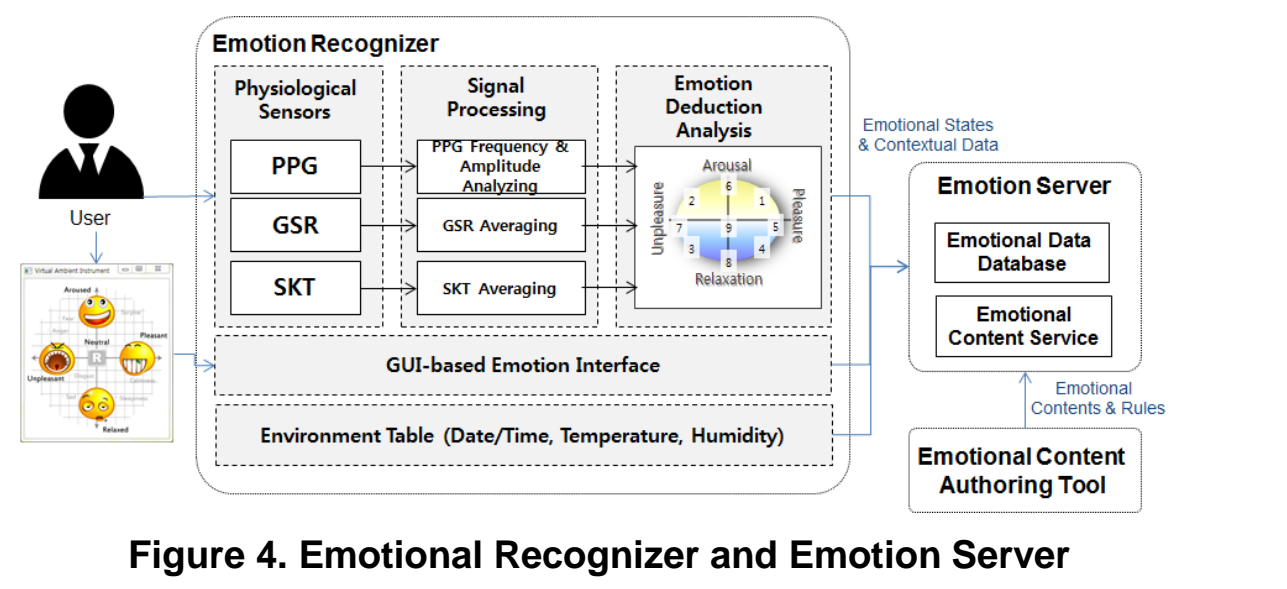

In this module, the emotion deduction algorithm infers user emotional states as arousal, distress, misery, depression, sleepiness, contentment, pleasure, excitement or neutral, based on the user's real-time physiological response [9]. Emotjon recognition is done either actively or passively as described in Kim's work [8]. Passive recognition analyzes the user's ANS physiological data and it is the primary method used in the EIC framework since it does not interrupt the user's task. Active recognition is also provided to complement the passive method. In active recognition, users cat explicitly specify their own emotional states by selecting one of nine emotion buttons on a graphical userinterface.

The Emotion Server is built on fop of a REST (Representational State Transfer)-based web service running on a Linux-based apache web server. The Emotion Server uploads the individual's raw physiological data and the determined emotional state in real-time. The server also stores user emotion data along with contextual information, such as date, time, location, humidity and temperature, for further analysis in the database. Moreover, it notifies EIC clients of the current user's amotional state. The Emotion Server also stores the individual's emotion wales and the emotion content elements that were created using the Emotion Content Authoring Module (Figure 5). In order to do this, each user is given a unique identificalion number along with his/her emotion data at given dates and times. Similarly, the emotion cule management database has also been created for each EIC client to handle customized features. The server also has default emotion rule sets defining how color, sound and light shouly be manipulated in the event that customized emotion rules do not exist for the user.

This framework uses the indirect mode by default. The indirect mode utilizes the user's accumulated emotion data, such as emotional states, emotion rules and emotion content elements, retrieved from the Emotion Server. It is implemented using the open source cURL library to communicate with the server's REST services. On the other hand, the direct mode supports the Emotion Communication Module to directly receive user emotion data from the Emotion Recognizer Module. It is implemented using the observer design pattern [22]. That is, the Emotion Communication Module registers itself into the Emotion Recognizer Module and gets notified whenever user emotional state changes.

\subsection{The Emotion Content Authoring Module}

As shown in Figure 5, the Emotion Content Authoring Module allows the user to create his/her own emotion expression rules that respond to emotional states. This module provides a graphical user interface to specify user-specific emotion rules, such as the manipulation of foreground and background color, light and sound. With this module, the user can customize 
the visual aspects of an image (for an emotion digital picture frame) or a texture map (for emotionally intelligent 3D games or virtual environments) in response to his/her emotional states. The user can also adjust the speed of the background music and sound effects and alter the brightness of the light and color within a 3D emotion content.

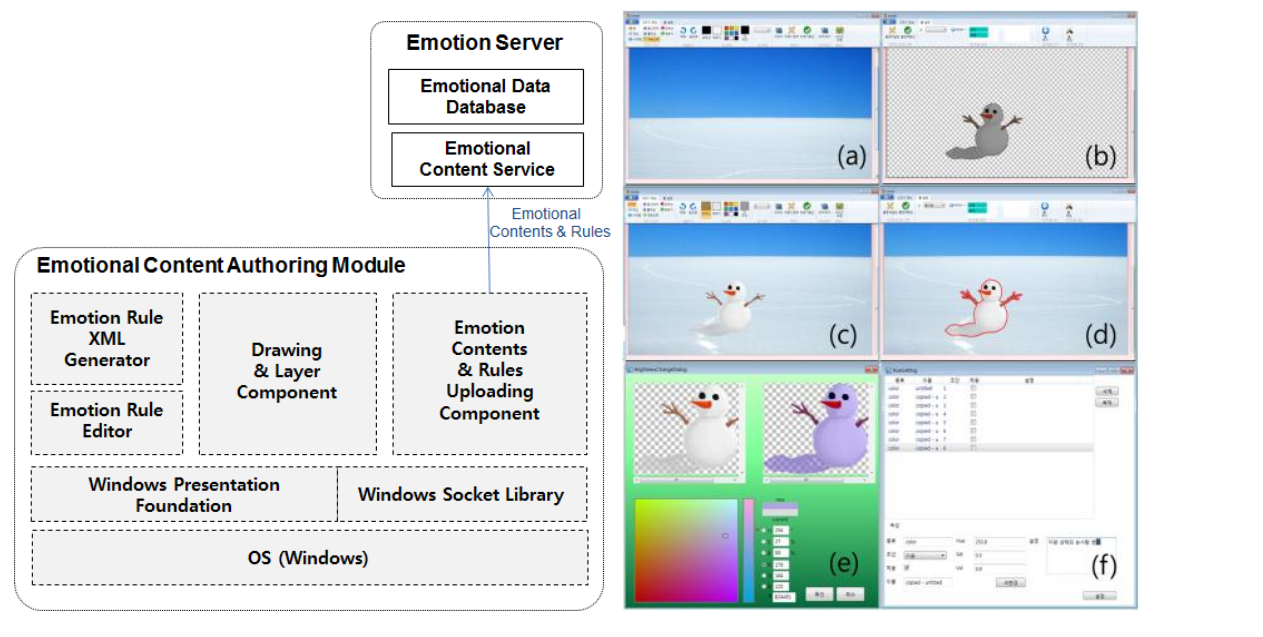

Figure 5. Emotion Content Authoring Module

This module is inspired by a $2 \mathrm{D}$ - paint drawing or picture editor program, like Adobe Photoshop. It provides much of the same basic image editing functionalities, including drawing primitive shapes, coloring, selecting objects, separation into different layers, etc. To create a new emotion rule, theyser starts by selecting a region, called the emotion rule region, in the workspace. The emotion rule region is the area where a user can alter color, saturation and lighting to reflect emotion expression. Once this region is selected, the user can specify an emotion rule bychoosing an embtion response from the toolbox. With this layer and region functionality, the user can create different emotion rules within the content.

Figure 5 shows a screenshot of creating an emotion rule using the Emotion Content Authoring Module. Figure 5(a) shows the original background photo of a continental glacier. Figure 5(b) shows a Snowman character created in a separate layer using the drawing component of the authorng tool. Figure 5(c) shows the snowman character layer being placed on top of the background photo and then merged into one layer. Figure 5(d) shows a region around the snowman being created to specify an emotion rule. Figure 5(e) shows the new color manipuation rule. Figure 5(f) shows the rule definition window used to change the color of the region. After an emotion rule is created, it saved in an XML file.

As shown in Figure 5, the emotional rules can be assigned to different layers. Similar to the layer concept in Adobe Photoshop, the emotion layer is defined as being a stackable but independent plane, in which users can draw any object and construct separate emotion rules. It is useful for organizing and managing the objects and accompanying emotion rules together. The layer is used to distinguish the foreground and background objects. It is also used to specify emotion rules on a particular field without affecting the overall look of the content. This feature makes for easier editing of emotion rules later on.

When the Emotion Content Authoring Module is first used, an identification number is given to each user for the purposes of storing his/her emotion rules and contents in the Emotion Server. When a user uploads a new emotion content (such as an image or a movie clip) to the server, the contents and emotion rules are stored separately and can be identified specifically. Other users can receive the Emotionally Intelligent Contents via URL access. 
This feature allows a user to specify his/her emotion rules without affecting other users' emotion rules.

\subsection{The Emotion Rule Controller Module}

As shown in Figure 6, the Emotion Rule Controller Module retrieves XML-based emotion rules from the server and parses them to construct a hierarchical structure of the emotion rules. When a new updated emotional state is received, it traverses the rule hierarchy to search for the appropriate emotion rule and then downloads the appropriate emotion contents and additional rules. Next, it generates emotion rendering commands to be sent to the Emotion Content Player Module to manipulate and render the contents based po the user's emotional state. Figure 6 shows an emotion rule XML file used in a three-dimensional emotional game called "Nocturnes Thief" [8]. The emotion rules are created using the Emotion Content Authoring Module. This emotion rule enables the changing of the texture map of a 3D model in the game. It contains the width and height infornhation of the texture map image and the file name. It also shows several nules that define certain color masks (actions) applied on some texture map areas (regions) for a/specific emotional state (condition).

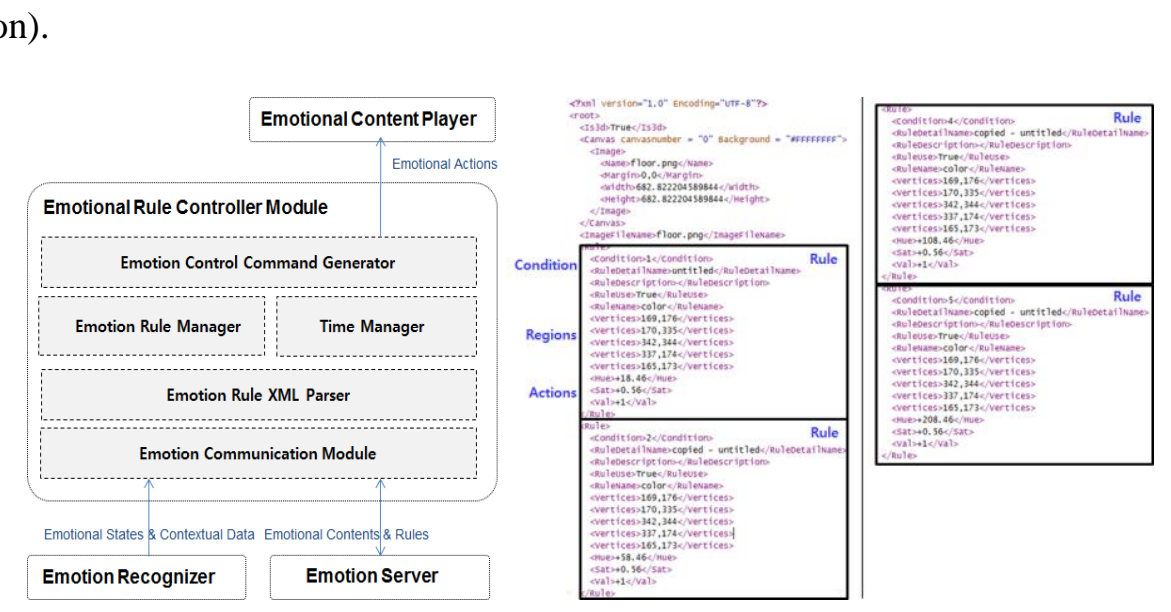

Figure 6. Emotion Rule Controller Module and XML-based Emotion Rules

\subsection{The Emotion Content Player Module}

The Emotion Content Player Module is responsible for playing EICs on various display devices, such as the iPhone, iPad, Android phone or desktop computer. EIC dynamically modifies its elements (such as the picture, texture images or sound) according to individual emotion rules specific to a given user's emotional state. This module checks whether new data is available on the Emotion Server. It then downloads new data upon the user's request and analyzes the data to respond to individual emotional states by changing the content's graphics and sounds according to the emotion rules. It also creates widgets on the screen and initializes 3D graphics rendering components. 

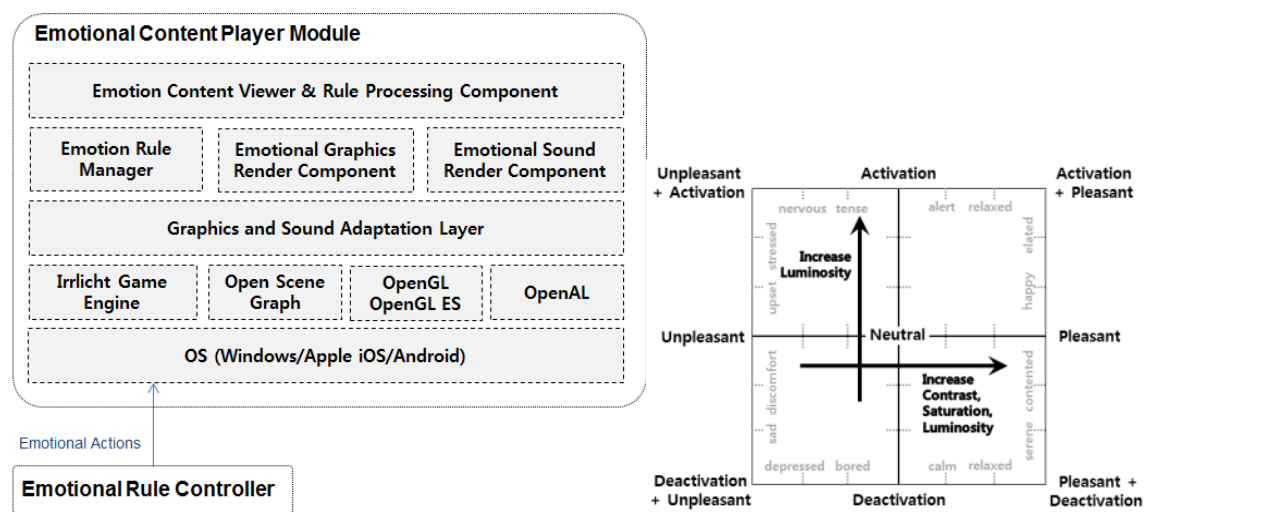

\section{Figure 7. Emotional Content Player Module and Default Color/Mask Model}

As shown in Figure 7, the Emotion Content Player Modwles are built on top of the Irrlicht three-dimensional game engine, the Open Scene Graph cene graph library, the OpenGL graphics library and the OpenAL sound library. It consists of a number of hybrid C++ interfaces to support texture and light management and manipulation utilizing various graphics and sound rendering engines. The Emotion Graphics Render Component handles the color and texture blending management ${ }^{\circ}$ or manipulating the texture map in real-time by putting emotion color masks on the original texture images. It also handles the color and brightness of lighting and fog effects to create thesambient mood and color tone. The Emotion Sound Renderer Component manages different kategories of music and sound, and it also controls volume and play speed. These high-level and abstract interfaces allow developers to build their contents to be emotion-aware without the need for much knowledge about the underlying platforms.

As shown in Figure 7 the default color mask model provides several sets of color palettes composed of nine different colors 48$]$ where brightness and color saturation increase as the emotion goes from sleepy to arous l. It is used to mask the color on the underlying images and texture maps depending en the user's emotional state. The color masking is done by first calculating the average REB color values of the image using Equation (1). Then, the final color is calculated by adding the color value and the difference between the default color mask value and the average color using Equation (2). This method preserves the tone, brightness and texture of the underlying materials of the original image even after the color mask is applied.

$$
\begin{aligned}
& \text { AogColor }=\left(\sum_{i=0}^{w-1} \sum_{j=0}^{h-1} \operatorname{Color}(i, j)\right) /(w \times h \times 255) \\
& \text { DestColor }(i, j)=\operatorname{Color}(i, j)+(\operatorname{ColorMask}-\text { AvgColor })
\end{aligned}
$$

The EIC framework also allows for the addition of an emotion avatar as a new character to represent the user's emotion or the replacement of it with one of existing objects in the Emotionally Intelligent Contents [8]. The emotion avatar (either a picture for a 2D content or a 3D model) can be retrieved from the server via a web service or loaded from local storage. The emotion avatar is packaged in a proprietary container file, which is a variant of the compressed zip file format. The compressed file contains a description of the embedded files in XML format, avatar files and some other emotion rules describing how the avatar's visual aesthetics should behave according to user emotion. 


\subsection{The Workflow of Emotionally Intelligent Contents}

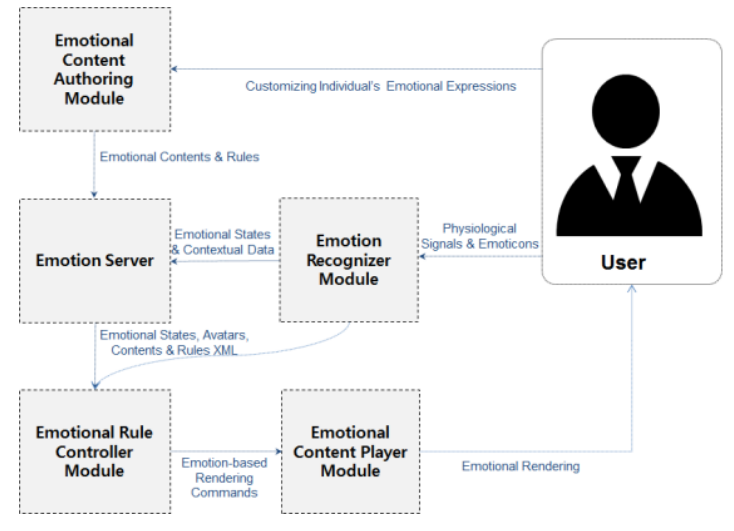

Figure 8. The Basic Workflow of Emotionally Intelligent Content

Figure 8 shows the basic workflow of Emotionaly Intelligent Contents:

- The EIC is started by a user who is wearing PPG, SKT, and GSR physiological sensors. The Emotion Recognizer Module analyzes the physiological signals and determines the individual's current emotional state.

- The individual's current emotional state is sent to the persistent database storage in the Emotion Server.

- The Emotion Rule Controller Module retrieves the individual's emotional state and the emotion rules via the Emption Communication Module. The emotion rules written in XML are parsed for the emotion rendering commands, which are then sent to the Emotion Content Player Module

- Also, the user can customze his/her emotion rules and contents using the Emotion Content Authoring Modale, which are then also sent to the Emotion Server. The newly updated emotion rules and contents are processed in the Emotion Rule Controller Module and applied in the Emotion Content Player Module.

- The Emotion Content Player Module renders the visual and aural properties of the contents by retrieving and applying the individual's emotional state and the emotion rules. If neeessary, it also downloads additional data, such as avatar 3D models, pictures, and texture map images.

- The process of changing EIC graphics and sounds may also affect the user's own emotional state. This change in emotional state is also sent to the Emotion Server and the whole process is started again.

\section{Applications}

We have built a few prototype applications using the EIC framework. These prototypes include the Emotion Video Player, the Emotion Nocturne Thief game, the EmOcean virtual environment and the Emotion Digital Picture Frame. 


\subsection{The Emotion Video Player}

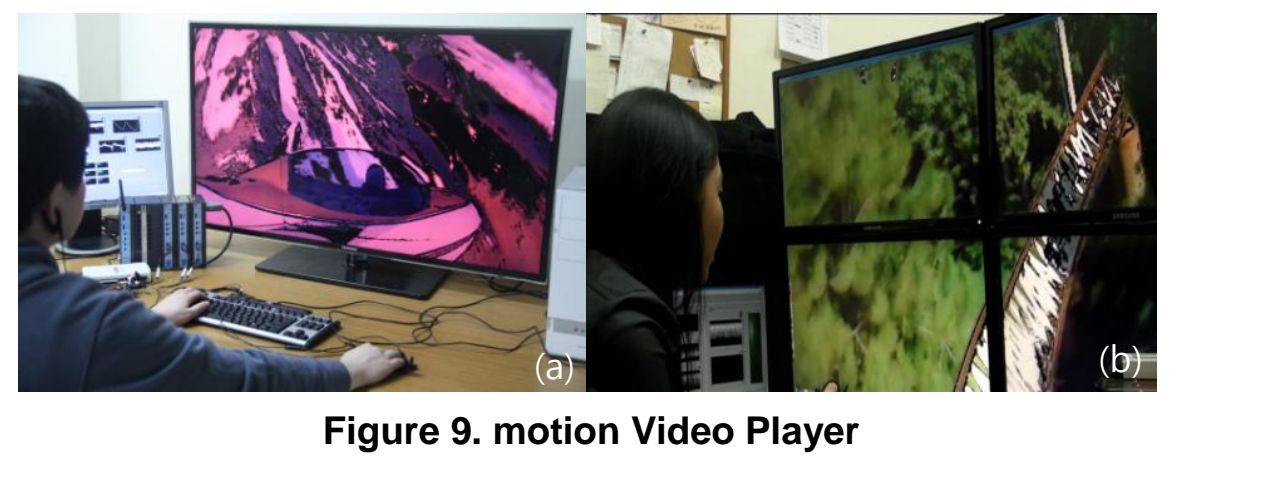

Figure 9 shows the Emotion Video Player, which manipulates the visual and aural aesthetics in real-time based on a user's emotional state. The Emotion Video Player can change the mood of the video footage and the tempo of the background music and sound effects according to user emotion. It is constructed using the EIC ramework, the Open Scene Graph graphics library and the DirectShow video library. When this application is started, it first loads the emotion rule for the viewer. It then applies the emotion color masking model, specified in the rule, to each frame of the move before it is rendered on the screen. It also manipulates the movie sounds at the sametime based or the user's emotion.

If no rule is specified, the default emotion color masking model (as shown in Figure 7) is used instead. Figure 9(a) shows the user watching a video clip using the Emotion Video Player. It shows how the color-masking model is applied to the whole video screen. The emotion rules can also be used to change only some portions of the movie frame instead of the whole screen. Figure $9(\mathrm{~b})$ shows how only the railroad areas of the roller coaster scene are affected by the emotion color-masking model. This feature of the Emotion Movie Player can be quite useful when-we want to eithey emphasize or deemphasize specific areas of the screen.

\subsection{The Emotion Nocturne Thief Game}

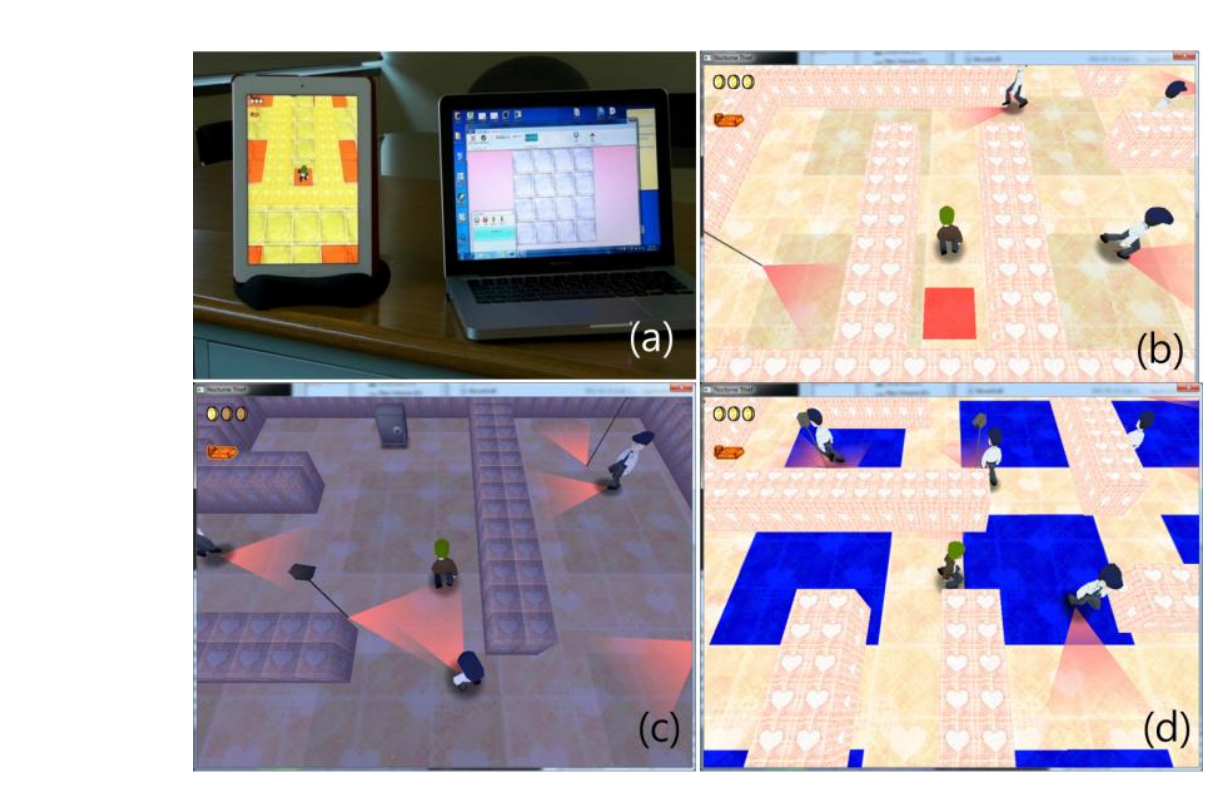

Figure 10. Emotion Nocturne Thief Game 
Figure 10 shows the Emotion Nocturne Thief game, which is an open-source game written using the Irrlicht game engine. The original game runs on Microsoft Windows and Linux. The object of the game is to get the thief character in the game to steal gold bars while avoiding the guards. We reconstructed this game to become emotion-aware using this EIC framework and also ported it to Apple's iOS platform (iPhone and iPad). Figure 10(a) shows a user constructing an emotion rule that would be used in the game using the Emotion Content Authoring Module. Figure 10(b) - (d) illustrates how the whole game world or just some portions of the texture maps can be manipulated as a user's emotional state changes.

Not only can the colors and sounds be changed in the Emotion Nocturne Thief game, the brightness, lighting, and texture maps of the 3D models within the game can also be modified based on the user's emotional states. For instance, the thief's (the emotion character in the game) clothes can be changed in real-time if an emotion rule is set. The EId framework enables the user to exchange this 3D character model with his/her emotion character, called an emotion avatar. This emotion avatar works exactly the same as the original game character, but now, however has been modified to reflect the emotional state of the player [8]. Since all the logistics of user emotion response is embedded in the EIC framework, no other game logic needs to be modified.

\subsection{The EmOcean Virtual Environment}
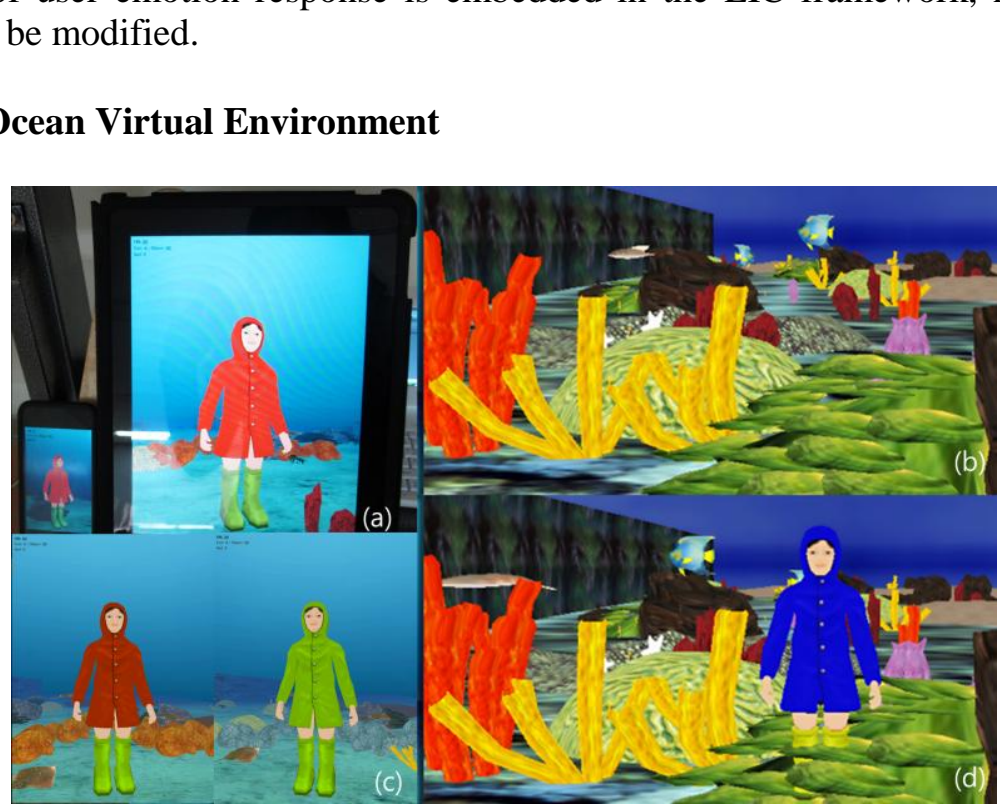

Figure 11. EmOcean Virtual Environment

Figure 11 shows EmOcean, an emotion-based virtual environment built using the EIC framework. This application runs on Microsoft Windows and the iOS platform. EmOcean allows a user to navigate under the sea, while the colors of the nearby fish, seaweed, and coral change colors in response to his/her emotion. Fog color, light color and brightness, and sound also change in response to the user's current emotion. The tempo and sound of the background music are also adjusted to reflect the user's emotional states and user-specified emotion rules.

Figure 11(a) and (b) show the EmOcean environment running on the iPod/iPad and the desktop computer, respectively. As was the case in the Emotion Nocturne Thief game, sharing of the emotion avatar in real-time is also possible. When the environment first appears, the program runs in first-person view mode as shown in Figure 11(b). The user can change to the view to third-person at run-time using a simple trigger as shown in Figure 11(c) 
and (d). As the user's emotional state changes, the color of the emotion avatar's clothes and the texture maps of the coral reefs in the environment also change.

\subsection{The Emotional Digital Picture Frame}
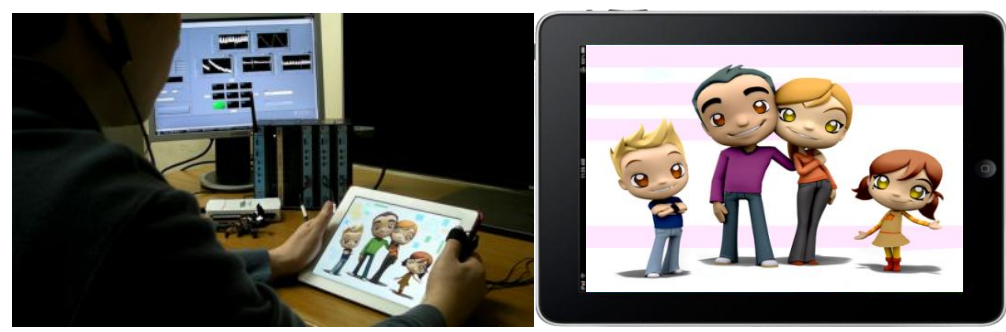

Figure 12. Emotion Digital Picture Frame

Figure 12 shows a picture of a family in the Emotion Digital Picture Frame developed on the Apple's iOS and Google's android operating system. This emotion picture frame system controls the pictures based on the emotion rules. An emotion ryle constructed using the Emotion Content Authoring Module specifies some regions of a picture and how these regions should be manipulated. Based on aser's emotion. the color of the father's and daughter's clothes is changed as are someportions of the background area.

The emotion digital picture frame also allows for the sharing of user's emotions with other remote users. The picture frame of the gne sending out the emotion recognizes user's emotion through physiological sensors and the status stored in the Emotion Server. Then, other users who are connected to the sender through the Emotion Server will receive information regarding the sender's emotional status in real-time. The receiver's picture frame software changes the visual properties of the pictures shown on his/her device to reflect the sender's emotional state. The receiver can also provide his/her own emotion rules to define how the emotion digital picture, frame should affect the picture.

\section{Evaluation of Intelligent Emotion Contents}

We conducted an experiment to evaluate differences in user experience between the original content and the Emotionally Intelligent Content, which dynamically changes in response to user emotion. Nine college and graduate school students participated in this experiment. The mean age of the subjects was 24.89 years old. First, the subjects attached the GSR, SKT and PPG physiological sensors to his/her fingers and ears for emotion recognition. In this experiment, subjects were first asked to watch the original movie twice for 3 minates on a 46" TV. After resting for 1 minute, subjects watched the Emotionally Intelligent Movie twice again for 3 minutes, to find out whether more changes would occur in the subjects' emotional state. During this experiment, changes in the subjects' emotional state were recorded every 0.25 seconds.

Table 1. The paired t-test results

\begin{tabular}{|l|c|c|}
\hline & $\mathrm{t}$ & Significance \\
\hline Comparison between the two original movies & 1.363655 & 0.172763 \\
\hline Comparison between the two emotional movies & 5.958713 & $2.79 \times 10-9$ \\
\hline $\begin{array}{l}\text { Comparison between the original movies and the } \\
\text { emotional movies }\end{array}$ & -2.11613 & 0.034396 \\
\hline
\end{tabular}


We evaluated changes in the subjects' emotional state while viewing the original movie and the Emotionally Intelligent Movie. First, we compared the variations in the emotional state between the two sequential viewings of the same content (i.e., between the two original contents and the two emotion contents). Then, we compared the changes in the subjects' emotional states between the viewings of the original and the emotion content. Table 1 shows the paired t-test results of the three conditions: for the two original contents, for the two emotion contents, and between the original and emotion content.

The paired t-test results for the viewings between the two original contents showed an average of 7.90 (standard deviation 3.49) and 8.03 (standard deviation 3.58). These results were not statistically significant. However, it was found that there was a significant difference between the viewings of the two emotion contents $(\mathrm{p}<0.05)$. Also, there was a significant difference $(\mathrm{p}<0.05)$ between the viewing of the original content and the emotion content, with the original content group having an average of 7.92 (standard deviation 4.30) and the emotion content group having an average of 8.36 (standard deviation 2.48). The results revealed that the Emotionally Intelligent Movie had a significant influence on the subjects' emotional states as compared to the original movie.

In a subjective post-test survey, subjects reported a great deal of interest in the Emotionally Intelligent Movie. The subjects especially showed a high interes in the Emotion Content Authoring Tool, which allows them to specify their owncemotion rules. However, some subjects indicated that applying individualized rules for emotion was somewhat inconvenient.

\section{Conclusion}

Emotion contents provide enjoyment and allow users to become more immersed in the contents. The Emotionally Intelligent Content supports dynamic changes of its visual and aural elements in real-time based on the aser's emotional state. It also allows for the unique expression of user emiotion based on the user's own emotion rules which define how the emotion contents react to user emotion. Moreover, EIC provides emotion-sharing capabilities by allowing users to replace the content object with their own emotion avatars or by adding special visual/aural effects at run-time in response to the user's emotional states.

In this research we proposed an EIC framework, which allows for easy construction of emotion contents, through the creation and expression of user-specific emotion rules, that can be shared with other users. The EIC framework consists of the Emotion Recognizer Module, the Emotion Server, the Emotion Content Authoring Module, the Emotion Rule Controller Module and the Emotion Content Player Module. The Emotion Recognizer assesses the user's emotiona state using ANS physiological sensors. The Emotion Server stores the user's emotional states, emotion rules, and emotion contents. The Emotion Content Authoring Modale allows users to define their own emotion rules for how the emotion contents would respond to their emotional states. The Emotion Rule Controller Module processes the user's emotional rules and sends commands to the Emotion Content Player Module to render the actual visual and aural aspects of the emotion contents.

In this paper we also presented a few emotion contents built using the EIC framework. The Emotion Content Authoring tool allows users to create emotion expression rules with individual preferences for color, brightness, and sound. The Emotion Video Player converts any video clip into emotionally intelligent footage. In the Emotion Nocturnes Thief game and EmOcean, the color and background music are dynamically changed according to user emotion and emotion avatars are added in real-time to represent the user's emotion without interrupting the user's experience. The emotion digital picture frame allows a number of remote users to share their emotions by changing the visual and aural properties of the pictures through the server. 
We also conducted an experiment comparing user experience while viewing the original contents and the Emotionally Intelligent Contents. The paired t-test result showed that there was a significant difference in user experience between viewing the original movie and the emotion movie, while there was no significant difference in user experience between two sequential viewings of the original movie. In the future, we plan to adapt Emotionally Intelligent Contents for educational purposes. This educational EIC would draw user attention when it appears that the user is getting bored. We will also conduct more systematic experiments to determine how emotion contents can be engaging or distracting for users depending on their emotional states.

\section{Acknowledgements}

This research was supported by Basic Science Research Progiam through the National Research Foundation of Korea (NRF) funded by the Ministry of Edycation, Science and Technology (2012R1A1A1010815), and a 2013 Research Grant from(Sangmyung University.

\section{References}

[1] R. Cowie, E. D. Cowie, N. Tspapatsoulis, G. Notsis, S. Kollians, W. Fellenz and J. G. Taylor, "Emotion Recognition in Human-Computer Interaction UEEE Signal Progessing Magazine, vol. 18, no. 1 (2002), pp. 32-80.

[2] G. Castellano, L. Kessous and G. CaridaRis "Emotion Recognition through Multiple Modalities: Face, Body, Gesture, Speech", Lecture Notesïn Computer Science, vol.4868 (2008), pp. 92-103.

[3] C. -Y. Chang, J. -S. Tsai, C. -J. Wang and P. -C. Chung, "Emotion Recognition with Consideration of Facial Expression and Physiological_Signals", Proceedings of the IEEE Symposium on Computational Intelligence in Bioinformatics and Computational Biology, (2009) March 30-April 2, Nashville, TN, U.S.A., pp. 278-283.

[4] Y. Wang and L Guan, "Recognizing Human Emotional State from Audiovisual Signals", IEEE Transactions on Multimedia, vol. 10, no, 4 (2008), pp. 659-668.

[5] W. Zhou, N. Xiang and X. Zhow, KTowards 3D Communications: Real Time Emotion Driven 3D Virtual Facial Animation, In Proceedings of the Workshop on Digital Media and Digital Content Management, (2011) May 15-17. Hangzhou China, pp. 132-135.

[6] V. Herrera, R. Miguel, J. J. Castro-Schez and C. Glez-Morcillo, "Using an Emotional Intelligent Agent to support customers' searches interactively in e-marketplaces", Proceedings of $22^{\text {nd }}$ International Conference on Tools with Artificial Intelligence, (2010) October 27-29, Arras, France, pp. 15-22.

[7] M. Kim, K. S. Pank and Y. Cho, "Design and Implementation of the iOS-based Game Framework for Emotional Character Expression”, Journal of The Korean Society for Computer Game, vol. 24, no. 1, (2011), pp. 63-72.

[8] M. Kim, K. S.Park, D. Kim and Y. Cho, "Emotional Intelligent Contents: Expressing User's Own Emotion within Contents", 10th International Conference on Entertainment Computing, Lecture Notes in Computer Science, yol. 6972, (2011), pp. 391-394.

[9] Km, J. H. Kim, E. C. Lee, M. C. Whang and Y. Cho, "Interactive emotional content communications system using portable wireless biofeedback device", IEEE Transactions on Consumer Electronics, vol. 57, no. 4, (2011), pp. 1929-1936.

[10] C. -L. Lin, P. -S. Gau, K. -J. Lai, Y. -K. Chu and C. -H. Chen, "Emotion Caster: Tangible Emotion Sharing Device and Multimedia Display Platform for Intuitive Interactions", Proceedings of the 13th IEEE International Symposium on Consumer Electronics (2009) January 10-14, Las Vegas, Nevada, U.S.A., pp. 988-989.

[11] D. -S. Kwon, Y. K. Kwak, J. C. Park, M. J. Chung, E. -S. Jee, K. -S. Park, H. -R. Kim, Y. -M. Kim, J. -C. Park, E. H. Kim, K. H. Hyun, H. -J. Min, H. S. Lee, J. W. Park, S. H. Jo, S. -Y. Park and K. -W Lee, "Emotion Interaction System for a Service Robot", Proceedings of the16th IEEE International Conference on Robot \& Human Interactive Communication, (2007) August 26-29, Jeju Island, Korea, pp. 351-356.

[12] J. Gonzalez-Sanchez, M. E. Chavez-Echeagaray, R. Atkinson and W. Burleson, "ABE: An Agent-Based Software Architecture for A Multimodal Emotion Recognition Framework", Proceedings of the 9th Working IEEE/IFIP Conference on Software Architecture, (2011) June 20-24, Boulder, Colorado, pp. 187-193. 
[13] H. Zhao, J. J. Zhang and S. McDougall, “Emotion-Driven Interactive Digital Storytelling," Lecture Notes in Computer Science, vol. 6972, (2007), pp. 22-27.

[14] D. L. Roberts, H. Narayanan and C. L. Isbell, "Learning to Influence Emotional Response for Interactive Storytelling", Proceedings of the AAAI Spring Symposium on Intelligent Narrative Technologies II, (2009) March 23-25, Standford, California, U.S.A., pp. 95-102.

[15] C. Jones and S. Jamie, "Acoustic Emotion Recognition for Affective Computer Gaming Affect and Emotion”, Lecture Note in Computer Science, vol. 4868, (2008), pp. 209-219.

[16] E. L. van den Broek, "Ubiquitous emotion-aware computing", Personal and Ubiquitous Computing, vol. 17, no. 1, (2013), pp. 53-67.

[17] J. H. Kim and E. Andre, "Emotion Recognition Based on Physiological Changes in Listening Music", IEEE Transactions on Pattern Analysis and Machine Intelligence, vol. 30, no. 12, (2008), pp. 2067-2083.

[18] J. A. Russell, "A circumplex model of affect", Journal of Personality and Social Psychology, vol. 39, no. 6, (1980), pp. 1161-1178.

[19] C. Y. Chang, J. Y. Zheng and C. J. Wang, "Based on Support Vector Regression for Emotion Reeognition using Physiological Signals", Proceedings of the International Joint Conference on Neyrat Ne works, (2010) July 18-23, Barcelona, Spain, pp. 1-7.

[20] M. C. Whang, J. S. Lim, K. R. Park, Y. Cho and W. Boucsein, “Are Computers Câpable of Understanding Our Emotional States?”, Lecture Notes in Computer Science, vol. 4562, (2008) pp. 204-211.

[21] M. S. Lee, J. S. Lim and M. C. Whang, "Controlling emotional state by sensitizing color paletts", Proceedings of the Fall Conference of Korean Society for Emotion \& Sensibility, (2010) November 19-20, Seoul, Korea, pp. 67-68.

[22] E. Gamma, R. Helm, R. Johnson and J. Vlissides, "Design Patterns: Elements of Reusable Object-Oriented Software," Addison Wesley, Massachusetts, (1995), pp 293-303.

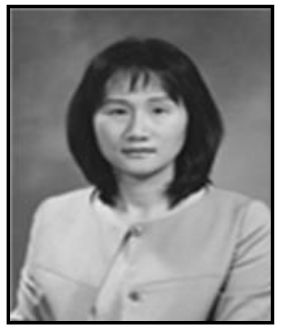

\section{Kyoung Shin Park}

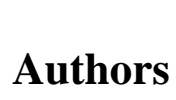

She 18 an associate professor at the Department of Multimedia Engineerng, Dankook Univerity in Korea. She acquired her Ph. D. in Comperter Science at the University of Illinois at Chicago (UIC) in 2003, with an emphasis in human computer interaction and graphics. She had also worked as a research professor at Digital Media Laboratory at Information \& Communications University until she joined at Dankook University and was a visiting scholar at MIT Media Laboratory in 2004. Herresearch interest includes human computer interaction, virtual reality, augmented reality, collaborative environments, tiled display systems, multimedia systems and 3D imaging.

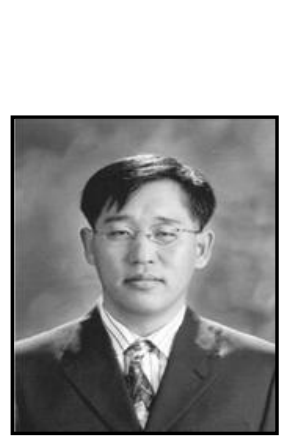

\section{Yongjoo Cho}

He is currently an associate professor at the Department of Digital Media, Sangmyung Univerity in Korea. He earned his Bachelor of Science in Computer Science at University of Illinois at UrbanaChampaign and Masters' of Science and Ph. D. degrees at University of Illinois at Chicago. His research interest includes Virtual Reality, 3D Display, Tiled Display System, Computer Supported Cooperative Work, and Virtual Learning Environments. 


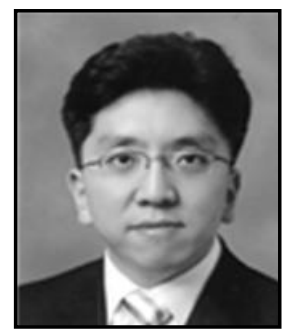

\section{Dong Keun Kim}

He received a B.S. degree in Information of Telecommunication from Sangmyung University in 2001, a M.S. degree in Medical Information Systems from Yonsei University in 2003, and a Ph.D. in Biomedical Engineering from Yonsei University in 2008. Currently, he serves as assistant professor in the Department of Digital Media at Sangmyung University, Seoul, Korea. His research interests include Multimedia System with Signal Processing, Human-Computer Interaction, and Healthcare System.

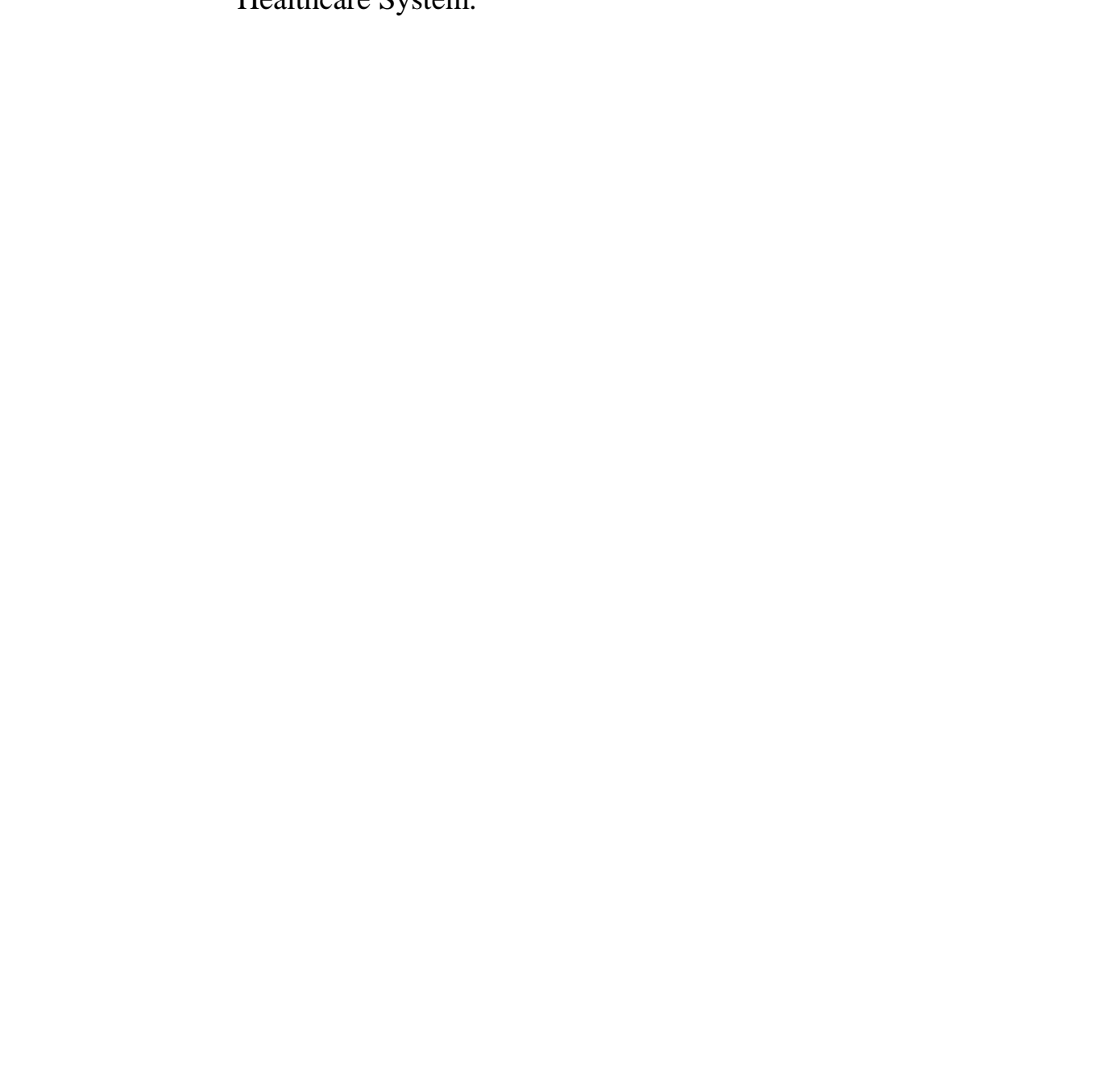


International Journal of Multimedia and Ubiquitous Engineering Vol.9, No.1 (2014)

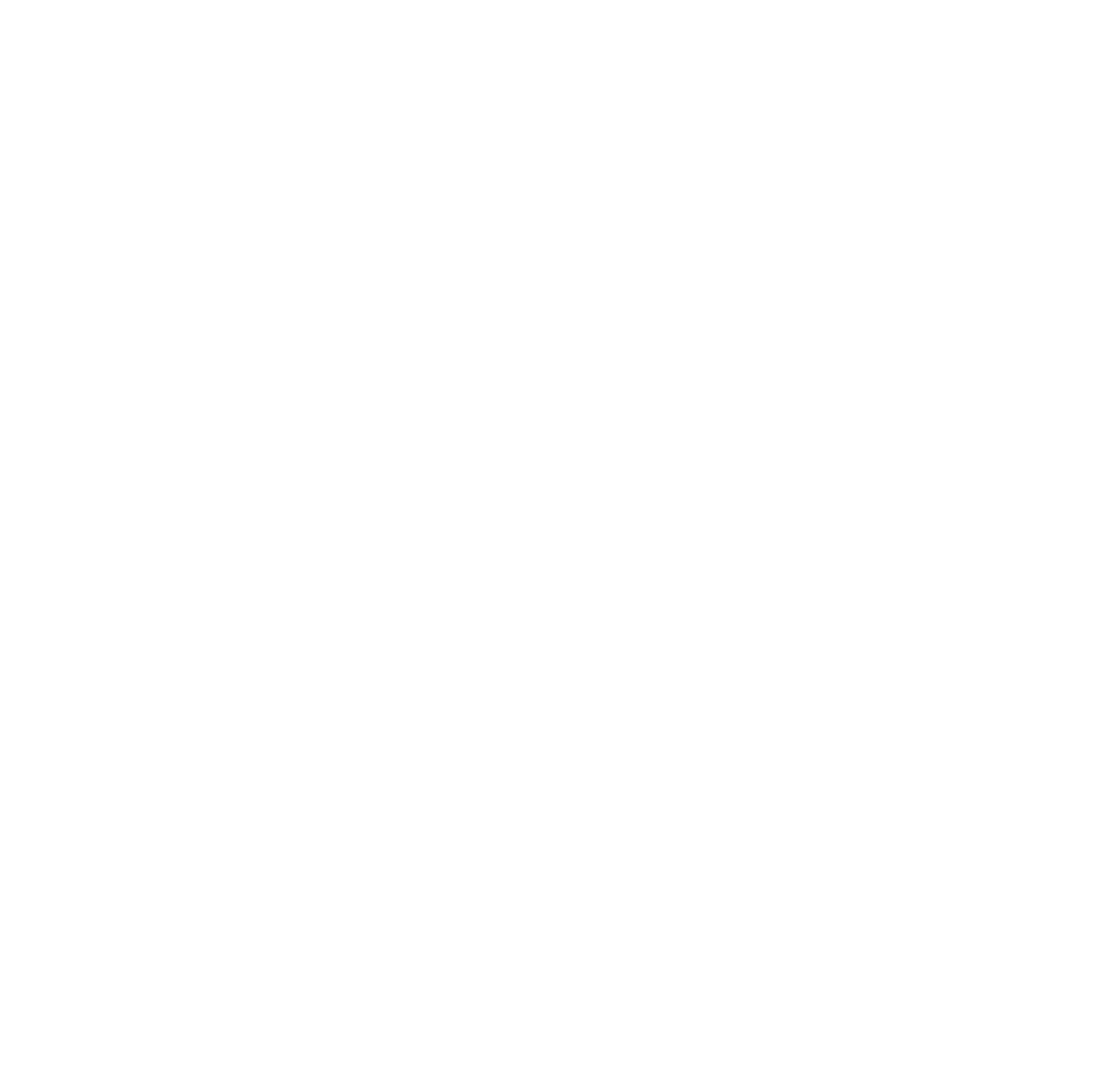

